\title{
Positron and positronium physics in atomic and molecular gases: Challenges for the $21^{\text {st }}$ century
}

\author{
F.A. Gianturco, Department of Chemistry, University of Rome "La Sapienza" and INFM, Rome, Italy
}

$T$ he subject of positron and positronium (Ps) physics with $I$ atoms and molecules is indeed one with a long history, but also a subject where a surprisingly large number of fundamental questions raised by a variety of recent observations still remain to be answered. Many of the problems in this area come up when discussing low-energy interaction of positrons and Ps with atomic and molecular aggregates and therefore provide the fundamental understanding for setting up a more quantitative approach to the chemistry of matter-antimatter processes.

To illustrate the status of modern research in positron physics and in the chemistry of positron/Ps processes, I have chosen to discuss only a few of the current issues which, hopefully, will convey to the reader the excitement coming from the resurgence of interest in this field and the current wave of experiments with their new findings. For a more extensive discussion that endeavours to better document the details of the topics which I shall only lightly touch on in this overview, I refer the interested reader to a recently published volume containing a broad range of articles on antimatter processes [1].

The increase in the interest of the scientific community on positrons as a research tool follows the development of low-energy positron beams, particularly over the last 15 years or so. It was realised early on, in fact, that to have such sources available might offer additional tests on the polarisation forces and scattering states probed by the counterpart studies with low energy electron beams of atomic and molecular gases. Furthermore, such studies indicated that correlation forces between the impinging positron and the bound electrons, either atomic or molecular, are much more important than the same effects in electron collisions, a consideration with profound implications in the sense that the virtual positronium $(\mathrm{Ps})$ formation channels (that can be viewed as transient bound state complexes moving in the field of the atomic or the molecular ion) now make the treatment of positron-atom/molecule collision events a much more complicated situation to handle with theoretical modellings. Thus, to have removed the non-locality of the exchange interaction that affects electron scattering processes turned out to be a simplification outweighted by the more complicated features of the electron-positron correlation effects which play a more explicit role, together with the one coming from the Ps formation channel. The latter is certainly one of the most fundamental examples of a charge exchange reaction that definitely requires for its description a quantum mechanical formulation of target-projectile interactions and dynamics. To this end, the new experimental techniques which exploit positron accumulators $[2,3]$ have markedly increased our capabilities for investigating positron and Ps interactions with matter at very low energies: they work already in the $\mathrm{meV}$ range and with possible developments which could bring us down to the $\mu \mathrm{eV}$ region. The elastic channels and the annihilation channels are always open as the kinetic energy of the probe approaches zero. Ps formation could also be energetically allowed whenever the target ionization energy is below $6.8 \mathrm{eV}$, the binding energy of the ground state Ps. Experiments could therefore be able to tell us what happens in that region of interaction where scattering states become close to the weakly bound states of the interacting species, if they exist at all. Both the annihilation process and the Ps formation cross section are expected, from general theoretical estimates, to increase proportionally to $1 / v$, $v$ being the relative velocity of the partners, as the energy tends to zero. One of the puzzling questions pertaining to such very low-energy scenario is provided by the possibility to find experimental evidence and theoretical confirmation on the existence of bound states or of metastable resonant states associated to cold positrons and Ps that are made to interact with fairly cold atomic and molecular gases. This is an aspect of the physics of antiparticles that has made very impressive progress, in the last 4-5 years, on the theoretical and computational predictions of such states while still rather absent at the experimental level. The definition of a stable antimatter compound could be had, in its simplest form, by changing a bound proton of a known stable compound with a positron. Thus $\mathrm{PsH}$ (positronium hydride) and $P_{s_{2}}$ become real chemical species that are related to the stable molecule of $\mathrm{H}_{2}$. Naturally, all such systems invariably annihilate and their lifetimes are of the order of $\sim 100$ picoseconds. Hence, an operational definition of a stable antimatter species could be had whenever the latter has at least one bound state that does not dissociate or autoionize before the inevitable annihilation occurs. Several of the properties of such apparently strange compounds are of direct interest at the fundamental level, besides being useful in many areas of applied, engineering studies [4]. Their binding energies, their annihilation rates and the momentum distributions of the emitted annihilation photons are amenable to calculations which have made tremendous progress in the last few years, thereby hopefull spurring future experimental attempts at producing comparable measurements: it may not

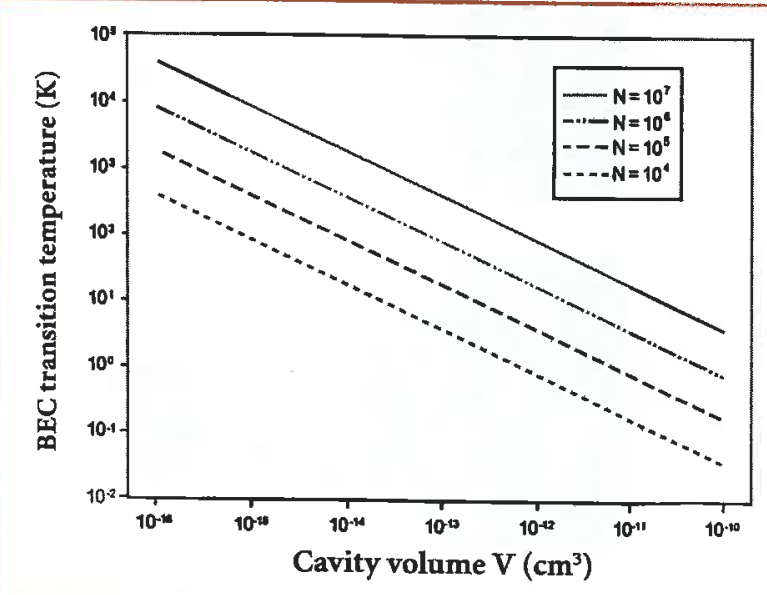

4. Fig. 1: The variation of the BEC transition temperature as a function of cavity sizes and for different, possible Ps densities in the cavity (adapted from ref. [14]). 
be long, therefore, before these will become available and will test the quality of the calculations.

Since the coupling between the quasi-discrete states and the continuum can be taken to be fairly weak [5], the calculations could then treat the latter states as conventional bound states and perform calculations of the pre-annihilation compounds using the quantum mechanical tools refined over the years to treat many-body bound structures $[6,7]$. Thus, the evaluation of correlation forces becomes an essential feature for establishing the likelyhood of producing positronic compounds which can be defined as stable. In the case of the Ps system, the formation of the neutral compounds like $\mathrm{PsX}$, with $\mathrm{X}$ being either an atomic or molecular partner, depends on its stability with respect to its main dissociation channel: $\mathrm{PsX} \rightarrow \mathrm{Ps}+\mathrm{X}$, hence one may qualitatively consider the source of binding to be due to simultaneous attraction of an electron to the positron and to the $\mathrm{X}$ complex. Theory therefore needs to provide a balanced evaluation of correlation forces versus annihilation rates before binding [6,7]. The most advanced efforts in this area have established that the presence of 'virtual' positronium formation during the interaction is one of the crucial ingredients for reliable values of binding energies vs annihilation rates [8]. In spite of the computational difficulties, in fact, more than 50 different antimatter compounds have been identified from theoretical studies, a truly explosive growth of the last four or five years [9].

It should have become already clear from the above examples that the renewed efforts in analysing low-energy behaviour of antimatter particles with ordinary matter compounds is providing one of the most exciting arenas in positron physics. It is therefore not surprising to discover that a large group of experimentalists, with strong theoretical support, is currently working on a cooperative effort named the ATHENA experiment [10]. The acronym describes an ApparaTus for High precision Experiments on Neutral Antimatter. It takes place at the Geneva CERN laboratory, carrying out experiments on antihydrogen $(\overline{\mathrm{H}})$ that are expected to test the CPT Invariance of Quantum Field Theory as well as Einstein's Equivalence Principle. The idea behind it is to trap $\mathrm{H}$ at very low temperatures of less than $1 \mathrm{~K}$ using an inhomogeneous magnetic field. The collaboration involves 7 different countries and more than fifty scientists. The above effort also tells us that our fundamental understanding of the mechanism by which the antiparticle will be kept in the trap and will avoid evaporative cooling via collisions with $\mathrm{H}, \mathrm{H}_{2}$ and $\mathrm{He}$ that are present in it, is an important requirement for choosing the best conditions which maximise the lifetime of $\bar{H}[11,12]$.

With the current resurgence of studies at ultra-low temperatures and ultra-low energies, antiparticles have also been considered as possible candidates for the production of Bose Einstein condensates (BEC) of bosonic Ps by studying the possibility of creating a dense gas of Ps particles in a microcavity within some solid state material which will then allow the possible phase transition to a BEC state of the ensemble [13]. We know already that such a transition occurs when an ensemble of low-T bosons undergoes the transition and collapse into a single, low-lying ground state of the global system. Because of the intrinsic instability of a matter-antimatter system (as is the one we are considering) and given the experimental difficulties for getting hold of low-energy positrons in sufficiently large quantities, such a possibility has so far received only limited attention and therefore we know very little on the properties of dense interacting Ps gases. The variation of the expected $\mathrm{BEC}$ transition temperatures as a function of the cavity volume, and for different required Ps densities, is shown in figure 1 and should give an idea of the hard

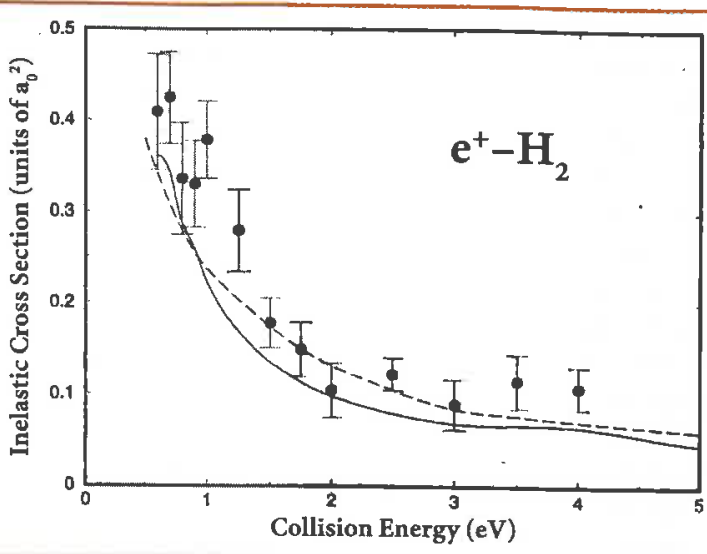

Aig. 2: Computed and measured partial integral cross Sections for the vibrational excitation of the $(\mathrm{O} \rightarrow 1)$ transition in the $\mathrm{H}_{2}$ molecule by positron collisions. The solid circles are the experiments from ref. [18] while the solid line reports recent theoretical calculations [23]. Both data are on an absolute scale without any adjustment (adapted from ref. [23]).

requirements for such exciting experiments to be successful [14]. They have been recently proposed to confine the Ps particles within microscopic cavities of solid sapphire that are located very near its surface, so that a highly focussed pulsed beam of energetic positrons can be made to impinge on the surface and be transported by injection into the cavity. After the Ps is formed there, and following the decay of its short-lived component, only the long-lived triplet state is expected to remain, thereby allowing the condensate to form during the cooling kinetics within the injected cavity $[14,15]$. Our further understanding of BEC physics means that issues like establishing some correlation between the BEC lifetime and the Ps own lifetime, the effect of impurities and of the confining waves on the condensate existence and behaviour, the Ps scattering length at ultra-low energies, the cross sections for elastic and reactive ( $\mathrm{Ps}_{2}$ formation) collisions will all be very important factors that will play a significant role in the experiments which will provide the above information for the first time. The creation, storage and manipulation of such special material under very extreme conditions as those proposed for the BEC experiments, will also require the combination of different expertise in the experimental and theoretical studies of atomic, molecular and solid state physics of the antimatter and will necessarily need the creation of a new generation of positron facilities.

The impact of new experimental facilities and of new sources of low-energy positrons has been particularly evident in the case of molecular gases and of the chemical processes which positron and positronium can activate within such gases [16-18]. Thus, absolute differential scattering cross sections and state-resolved inelastic scattering cross sections has become a reality with the recent development of high resolution $(\sim 20 \mathrm{meV})$, high brightness beam of positrons [19], produced by using a Penning trap to form a reservoir of cold positrons to be released in a controlled fashion [16]. One can therefore nowadays collect much more accurate data on inelastic processes in molecular gases, e.g. vibrational excitations of specific molecular modes and variations of the corresponding angular distributions of the scattered positrons as different modes are being excited, ionization cross sections for positron impact on atomic and molecular gases, both as a direct ionization process and as ionization with Ps formation $[20,21]$. In the latter cases, features related to a new 
process that has become known as electron capture to the continuum (ECC) have been identified [22], providing new challenges for the theoretical interpretation of such events even in simple atomic gases. An interesting example of how the new class of experiments could be met by theoretical comparison is shown in figure 2. We report there the integral, partial inelastic cross section fort the excitation of the $(0 \rightarrow 1)$ vibrational mode of the $\mathrm{H}_{2}$ molecule by positron impact. The experimental data are on an absolute scale [18] and the solid line calculations [23] are clearly reproducing experiments very closely and without any adjusted scaling.

Perhaps one of the most interesting molecular effects that has been uncovered by the recent experiments involving slow positrons interacting with polyatomic gases has been the behaviour of their annihilation rates in such gases [24]. The measured annihilation rates are found to be a linear function of the test gas pressure and the slope linearity yields the value of the rate, termed $Z_{\text {eff. }}$ The extremely broad range of observed $Z_{\text {eff }}$ values over the set of molecular gases which have been experimentally studied [25] indeed provides one of the challenges of positron physics that calls on the development of powerful computational models for cases where fairly complicated polyatomic gases are involved [25]. To make this point more evident, we report in figure 3 the behaviour of the $Z_{\text {eff }} / Z$ ratios for a wide variety of molecular species and as a function of $Z$. One clearly sees that the ratios are very far from unit when more complicated molecules are considered [26] and vary over several orders of magnitude.

The microscopic nature of $e^{+}-e^{-}$interactions with atoms and molecules is studied by measuring the Doppler-broadening of the $511-\mathrm{keV}$ annihilation gamma-ray line [24]. The Doppler line width is determined by the momentum distribution of the electrons which take part in the annihilation. The usual interpretation of this feature is that the positron has a relatively long de Broglie wavelength in the vicinity of the target, due to its net repulsive interaction with the electronuclear network of bound particles which make up the molecule. Consequently, the positron interacts with roughly the same probability with any of the valence electrons. However, the observation of $\mathrm{Z}_{\text {eff }}$ values which range from $\sim 20$ up to $10^{7}-10^{8}$ depending on the molecular gas points instead to the existence of qualitative differences between the mechanisms which are responsible for the annihilation in the atomic gases and in molecules of increasing complexity. To date, there has been no satisfactory explanation for such dramatic changes in molecular gases, beyond speculating on the formation of positron-molecule complexes with the existence of bound states depending on the molecular vibrational structure and with the occurrence of resonant mechanisms to enhance annihilation efficiency [27]. Such interesting conjecture has indeed made a number of predictions which are in qualitative agreement with the experiments and has therefore triggered both a flurry of more rigorous theoretical treatments and the gathering of additional experimental data. Both aspects of the problem are very hard to study: the experiments on the molecular gases require resolving the vibrational energy loss spectra for several modes of the molecule and to measure at those energy values the behaviour of the annihilation rates [28]. The theoretical models, on the other hand, need to treat as realistically as possible the dynamical couplings which take place between the impinging positron and the molecular vibrational modes during the annihilation process [29] and to do so for the large polyatomic species of the data in fig. 3 . However, the puzzle constitutes a very interesting test of our understanding of molecular processes at the nanoscopic level and extends the use of our computational tools into the area of antimatter-matter processes.

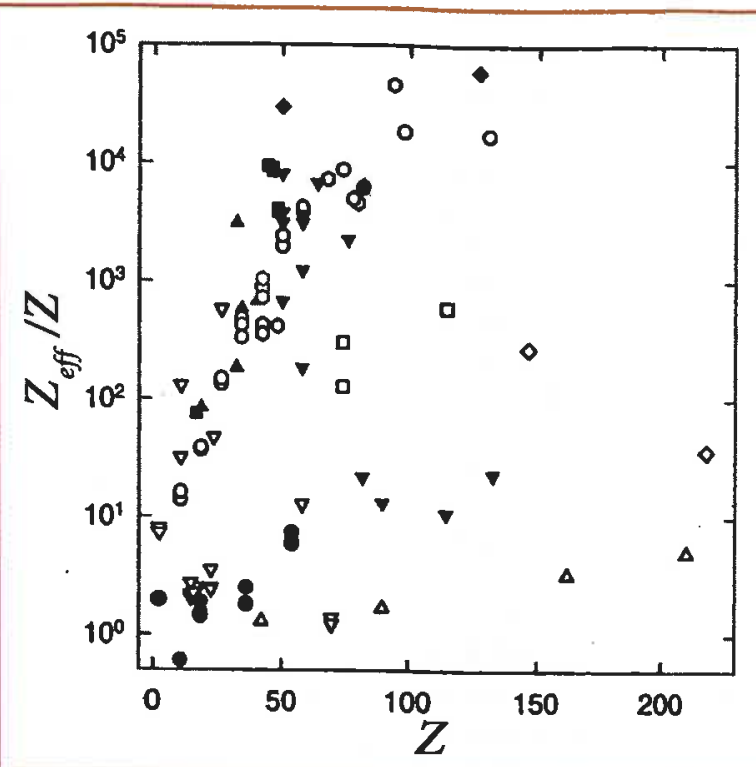

$\Delta$ Fig. 3: Experimental values of $Z_{\text {eff }} / Z$ plotted against $Z$, illustrating the fact that this quantity varies by orders of magnitude for modest changes in chemical species: $(\bullet)$ noble gases, $(\nabla)$ simple molecules $(0)$ alkanes, $(\Delta)$ perfluorinated alkanes, $(\square)$ perchlorinated alkanes, $(\diamond)$ perbrominated and periodated alkanes, ( $(\mathbf{n})$ alkenes, ( $\Delta$ ) oxygencontaining hydrocarbons, $(O)$ ring hydrocarbons, $(\nabla)$ substituted benzenes, and ( $\bullet$ ) large organic molecules (adapted from ref. [26]).

In conclusion, what I have tried to outline above should prove to the reader that the field of positron and positronium research is undergoing a remarkable revival: while the use of positrons in chemistry and physics, especially in the applied areas related to the above subjects like, for example, polymer studies, material analysis and surface studies, has been in existence for decades, it is fair to say that the use of low-energy positron sources under highresolution conditions is a much less mature field. The results gathered thus far are certainly promising and the response of the theoretical community has also been very encouraging in the sense that new quantum formulations and new computational models are being set up and refined to answer the experimental challenges both of the present and of the future observations. It is also not too far-fetched to expect that we could become capable of harnessing the annihilation photon energy produced by positronmatter interaction and put it to good use in many areas of our daily life. Such developments do point to the fact that antimatter will indeed matter a great deal for the science of the $21^{\text {st }}$ century.

\section{Acknowledgements}

The authors acknowledges the numerous fruitful and illuminating discussions on the subject of positron/Ps physics with molecular systems that have taken place over the years with several colleagues: T. Nishimura, T. Mukherjee, A. Occhigrossi, R.R. Lucchese, R. Curik, C.M. Surko, D.M. Schrader, A.S. Gosh, G. Gribakin, M.A.P. Lima, M. Kimura and I. Shimamura.

\section{References}

[1] C.M. Suko and F.A. Gianturco, Ed.s, New Directions in Antimatter Chemistry and Physics, Kluwer Academic Publishers, Dordrecht, The Netherlands (2001)

[2] R.G. Greaves, M.D. Tinkle and C.M. Surko, Phys. Plasma 1, 1439 (1994) 
[3] see also: M. Charlton and J.W. Humbertson, Positron Physics (Cambridge University Press) Cambridge, UK (2001)

[4] e.g. see: Positron Annihilation (P.C. Jain, R.M. Singu and K.P. Gopinathan, Ed.s) World Scientific, Singapore (1985)

[5] J.A. Wheeler, Ann. N.Y.Acad. Sci. 48, 219 (1946)

[6] J.Mitroy, M.W.J. Bromley and G.G. Ryzhilkh, Phys. Rev. Lett. 79, 4124 (1992)

[7] D. Bressanini, M. Mella and G. Morosi, J. Chem. Phys, 109, 1716 (1998)

[8] for a more detailed discussion, see all the contributions to Section III of ref. [1] (2001)

[9] e.g. see: D.M. Schrader and J. Moxom, chapt. 15 in ref. [1] (2001)

[10] M.H. Holzscheiter and M. Charlton, Rep. Progr. Phys. 62, 1 (1999)

[11] P. Froelich et al. Phis. Rev. Lett. 84, 4777 (2000)

[12] E.A.G.Armour et al., J. Phys. B 31, L679 (1998)

[13] e.g. see: P.M. Platzman and A.P. Mills Jr., Phys. Rev. B 49, 454 (1994)

[14] D.B. Cassidy and Golovchenko in: ref. [1],pg. 83 (2001)

[15] H. Saito and T. Hyodo, in ref. [1],pg. 101 (2001)

[16] S.J. Gilbert, R.G. Graeves and C.M. Surko, Appl. Phys. Lett. 470, 1944 (1997)
[17] S.J. Gilbert, J.P. Sullivan, R.G. Graeves and C.M. Suko, Nucl. Inst. and Meth. B171,81 (2000)

[18] J.P. Sullivan, S.J. Gilbert and C.M. Surko, Phys. Rev. Lett. 86, 128, 1494 (2001)

[19] S.J. Gilbert, C. Kurz, R.G. Graeves and C.M. Surko, Nucl. Inst. and Meth. B143, 188 (1998)

[20] J. Moxom, D.M. Schrader, G. Laricchia, Jun Xu and L.D. Hulet, Phys. Rev. A62, 052708 (1999)

[21] P. Ashley, J. Moxom and G. Laricchia, Phys. Rev. Lett. 77, 1250 (1996)

[22] A. Kover, G. Laricchia and R. Finch, Nucl. Inst. and Meth. B171, 103 (2000)

[23] F.A. Gianturco and T. Mukherjee, Phys. Rev. A64, 024703 (2001)

[24] e.g. see: T.J. Murphy and C.M. Surko, Phys. Rev. Lett. 67, 2954 (1991)

[25] K. Iwata, R.G. Graeves, T.J. Murphy et al. Phys. Rev. A51, 473 (1995)

[26] C.M. Surko in ref. [1], pg. 345 (2001)

[27] G.F. Gribakin, Phys. Rev. A61,22720 (2000)

[28] S.J. Gilbert, L.D. Barnes, J.P. Sullivan and C.M. Surko, Phys, Rev. Lett. 88, 043201-1 (2002)

[29] F.A. Gianturco and T. Mukherjee, Europhys. Lett. 48, 255 (1999). 